JOURNAL OF SYNCHROTRON RADIATION

ISSN 1600-5775

Received 24 January 2016

Accepted 18 May 2016

Edited by S. M. Heald, Argonne National Laboratory, USA

Keywords: full multiple scattering theory; V-bearing compounds; XANES; non-structural parameters.

Supporting information: this article has supporting information at journals.iucr.org/s

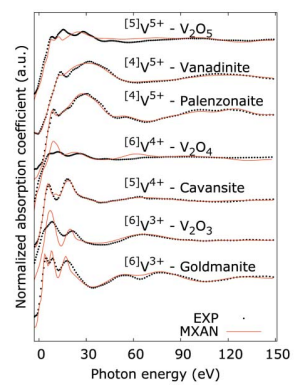

C 2016 International Union of Crystallography

\section{Vanadium $K$-edge XANES in vanadium-bearing model compounds: a full multiple scattering study}

\author{
Federico Benzi, ${ }^{a *}$ Gabriele Giuli, ${ }^{a}$ Stefano Della Longa ${ }^{b}$ and Eleonora Paris ${ }^{a}$

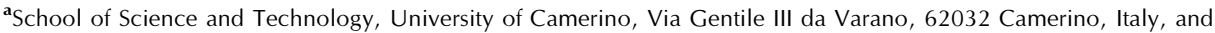 \\ b Dipartimento di Medicina Clinica, Sanità Pubblica, Scienze della Vita e dell'Ambiente, Università dell'Aquila, \\ Piazzale S. Tommasi 1, 67100 Coppito (AQ), Italy. *Correspondence e-mail: federico.benzi@unicam.it
}

A systematic study is presented on a set of vanadium-bearing model compounds, representative of the most common $\mathrm{V}$ coordination geometries and oxidation states, analysed by means of vanadium $K$-edge X-ray absorption near-edge spectroscopy calculations in the full multiple scattering (FMS) framework. Analysis and calibration of the free parameters of the theory under the muffintin approximation (muffin-tin overlap and interstitial potential) have been carried out by fitting the experimental spectra using the $M X A N$ program. The analysis shows a correlation of the fit parameters with the $\mathrm{V}$ coordination geometry and oxidation state. By making use of this correlation it is possible to approach the study of unknown V-bearing compounds with useful preliminary information.

\section{Introduction}

Vanadium is a transition metal that can occur in nature in a variety of oxidation states (from 0 to $5+$ ) and coordination geometries, with tetrahedral, square pyramidal and octahedral being the most common (Schindler et al., 2000). The $\mathrm{V}$ structural role is widely studied both in crystalline structures and in glasses, because V-bearing compounds and alloys find several applications in the steel industry, for catalysis (Hävecker et al., 2002; Surnev et al., 2003; Safonova et al., 2009; Walter et al., 2010) and as materials for cathodes in Li-ion batteries (Sakurai \& Yamaki, 1988; Katoh et al., 2010; Moretti et al., 2013). V-bearing glasses have been largely studied in the past 40 years because of their conductivity properties (Munakata, 1960; Mott, 1968; Sayer et al., 1971; Frazier \& France, 1977; Giuli et al., 2004; Seshasayee \& Muruganandam, 1998; McGreevy, 2001; Ori et al., 2011; Schuch et al., 2012). Furthermore, owing to the variety of oxidation states and coordination geometries, $\mathrm{V}$ model compounds can be a good choice to test the capabilities and drawbacks of codes for the calculation of theoretical XANES spectra.

The X-ray absorption near-edge spectroscopy (XANES) part of the X-ray absorption spectroscopy (XAS) technique (Bunker, 2010; Lee \& Pendry, 1975; Lee et al., 1981; Rehr \& Ankudinov, 2005; Bordiga et al., 2013) has long been exploited to obtain qualitative information using empirical or fingerprint methods. The empirical methods try to relate the spectral shape and the edge position to the absorbing atom local structure and oxidation state; the fingerprint methods compare some spectral features (mainly the pre-edge feature) of model compounds with known structure with the same features of an unknown compound to gather some quantitative information on the absorbing atom oxidation state and/or coordination geometry. Theoretical $a b$ initio calculations, in 
Table 1

V-bearing model compounds review of crystallographic data.

In ${ }^{[x]} \mathrm{V}^{y}, x$ represents $\mathrm{V}$ coordination geometry and $y$ represents $\mathrm{V}$ formal valence. Reference gives the source of the $\mathrm{CIF}$ file. $\langle\mathrm{V}-\mathrm{O}\rangle$ represents the average of the bond distances for the first $\mathrm{V}-\mathrm{O}$ coordination shells.

\begin{tabular}{llllll}
\hline Compound & $\mathrm{V}$ type & Formula & Space group & $\langle\mathrm{V}-\mathrm{O}\rangle(\AA)$ & Reference \\
\hline Goldmanite & ${ }^{[6]} \mathrm{V}^{3+}$ & $(\mathrm{Ca}, \mathrm{Mn})_{3}(\mathrm{~V}, \mathrm{Al}, \mathrm{Cr})_{2}\left(\mathrm{SiO}_{4}\right)_{3}$ & $I a 3 d$ & 2.018 & Righter et al. $(2011)$ \\
$\mathrm{V}_{2} \mathrm{O}_{3}$ & ${ }^{[6]} \mathrm{V}^{3+}$ & $\mathrm{V}_{2} \mathrm{O}_{3}$ & $R \overline{3} c$ & 2.007 & Finger \& Hazen $(1980)$ \\
Cavansite & ${ }^{[5]} \mathrm{V}^{4+}$ & $\mathrm{Ca}(\mathrm{VO}) \mathrm{Si}_{4} \mathrm{O}_{10} \cdot 4 \mathrm{H}_{2} \mathrm{O}$ & $P c m n$ & 1.913 & Danisi et al. $(2012)$ \\
$\mathrm{V}_{2} \mathrm{O}_{4}$ & ${ }^{[6]} \mathrm{V}^{4+}$ & $\mathrm{V}_{2} \mathrm{O}_{4}$ & $P 2_{1} / c$ & Rogers $(1993)$ \\
Palenzonaite & ${ }^{[4]} \mathrm{V}^{5+}$ & $\left(\mathrm{Ca}, \mathrm{Na}_{3} \mathrm{Mn}_{2}\left(\mathrm{~V}, \mathrm{As}, \mathrm{Si}_{3} \mathrm{O}_{12}\right.\right.$ & $I a 3 d$ & 1.94 & Nagashima \& Armbruster $(2012)$ \\
Vanadinite & ${ }^{[4]} \mathrm{V}^{5+}$ & $\mathrm{Pb}_{5}\left(\mathrm{VO}_{4}\right)_{3} \mathrm{Cl}$ & $P 6_{3} / m$ & 1.714 & Laufek et al. $(2006)$ \\
$\mathrm{V}_{2} \mathrm{O}_{5}$ & ${ }^{[5]} \mathrm{V}^{5+}$ & $\mathrm{V}_{2} \mathrm{O}_{5}$ & $P m m n$ & 1.710 & Shklover et al. $(1996)$ \\
\hline
\end{tabular}

principle, allow detailed information to be gathered on both the electronic properties and the structural information of the photoabsorber without any prior knowledge. The improvements in computing power and better approximations allowed us to successfully reproduce XANES spectra in several systems in order to obtain quantitative information about the local geometry and electronic properties of the target element (Benfatto \& Della Longa, 2001; Taillefumier et al., 2002; Prado \& Flank, 2005; Trcera et al., 2009; Nakanishi \& Ohta, 2009; Cabaret et al., 2010; Bordage et al., 2010). Among the approximations used to calculate the theoretical XANES spectra, full multiple scattering (FMS) theory in the muffin-tin (MT) approximation (Natoli \& Benfatto, 1986) is probably the most widely used, and it has been extensively used to investigate the local structure of a number of systems.

Several studies are available on the structural role of $\mathrm{V}$ in natural and synthetic samples (both on crystalline structures and in glasses) by means of XANES but most of them were focused on using fingerprint methods (Wong et al., 1984; Stizza et al., 1986; Poumellec et al., 1987; Giuli et al., 2004; Sutton et al., 2005; Faiz et al., 2007; Kavner et al., 2007; Chaurand et al., 2007). These methods are very useful for obtaining quantitative information about the $\mathrm{V}$ oxidation state and its coordination geometry, but they are unable to provide details about the bond distortion and coordination over the first shell. Some studies with theoretical modeling of XANES for V-bearing compounds are available in the literature (Bordage et al., 2010), but to our knowledge a systematic study on a variety of $\mathrm{V}$ model compounds with different oxidation states and coordination geometries is still lacking.

In this article we present a systematic study of the $\mathrm{V} K$-edge XANES spectra of a series of V-bearing model compounds in the full multiple scattering (FMS) framework. The purposes of this work are (i) to investigate the reliability of the FMS theory in calculating the $\mathrm{V} K$-edge XANES spectra of compounds with different $\mathrm{V}$ local environment (V coordination geometry and oxidation state) and (ii) to obtain information about non-structural parameters and observe their trends as a function of $\mathrm{V}$ coordination geometry and oxidation state in the model compounds. By fitting non-structural parameters (such as muffin-tin overlap and interstitial potential) to the spectra of well characterized $\mathrm{V}$ model compounds, it is possible to approach the study of unknown V-bearing compounds using a set of non-physical parameters which have already been proven to be reliable. The use of these starting values for such parameters can help in greatly reducing the complexity in the preliminary steps of XANES calculations when studying complex systems for which poor structural information is available.

\section{Materials and methods}

A set of V-bearing model compounds has been chosen to represent the most common $\mathrm{V}$ coordination geometry and oxidation states (Wong et al., 1984; Schindler et al., 2000; Giuli et al., 2004). The model compounds (Table 1) are: (i) $\mathrm{V}_{2} \mathrm{O}_{3}$ and goldmanite for $\mathrm{V}^{3+}$ in octahedral coordination $\left({ }^{[6]} \mathrm{V}^{3+}\right)$; (ii) cavansite for $\mathrm{V}^{4+}$ in square pyramidal coordination $\left({ }^{[5]} \mathrm{V}^{4+}\right)$; (iii) $\mathrm{V}_{2} \mathrm{O}_{4}$ for $\mathrm{V}^{4+}$ in octahedral coordination $\left({ }^{[6]} \mathrm{V}^{4+}\right)$; (iv) palenzonaite and vanadinite for $\mathrm{V}^{5+}$ in tetrahedral coordination $\left({ }^{[4]} \mathrm{V}^{5+}\right)$ and $(\mathrm{v}) \mathrm{V}_{2} \mathrm{O}_{5}$ for $\mathrm{V}^{5+}$ in square pyramidal coordination $\left({ }^{[5]} \mathrm{V}^{5+}\right) . \quad \mathrm{V}_{2} \mathrm{O}_{3}, \quad \mathrm{~V}_{2} \mathrm{O}_{4}$ and $\mathrm{V}_{2} \mathrm{O}_{5}$ are synthetic compounds (powders), whereas the others are natural samples. The natural standards were separated by hand picking small fragments from thumb-sized crystals and choosing the clearest portions to avoid impurities, except for goldmanite which was found as approximately 200 to $400 \mu \mathrm{m}$ transparent crystals. All samples were checked for purity by optical microscopy, X-ray diffraction and scanning electron microscopy. For each model compound a recent X-ray diffraction structural refinement present in the literature is chosen to build the starting cluster to calculate the theoretical XANES spectra.

Goldmanite, cavansite, palenzonaite and vanadinite were finely ground and deposited on Kapton tape. $\mathrm{V}_{2} \mathrm{O}_{3}, \mathrm{~V}_{2} \mathrm{O}_{4}$ and $\mathrm{V}_{2} \mathrm{O}_{5}$ samples came from reagent grade powders and they were prepared in a cellulose-based pellet. The spectra have been collected during different experiments: (i) goldmanite, cavansite and vanadinite spectra were collected around the $\mathrm{V}$ $K$-edge $(5465 \mathrm{eV})$ at the GILDA CRG beamline (d'Acapito et al., 1998) of the European Synchrotron Radiation Facility (ESRF) in Grenoble, France. The storage ring operated at $6 \mathrm{GeV}$ in $7 / 8+1$ filling mode with a maximum electron current of $200 \mathrm{~mA}$. The monochromator was equipped with a pair of $\mathrm{Si}(111)$ crystals and it was run in dynamical focusing mode (Pascarelli et al., 1996). Harmonic rejection was achieved by using a pair of Pt-coated mirrors with a cutoff energy of $26 \mathrm{keV}$. The incident beam was measured with an ionization 
chamber and the fluorescence signal was collected with a highpurity 13 -element Ge detector. (ii) A palenzonaite spectrum was collected at the ID26 beamline (Gauthier et al., 1999) at ESRF. The storage ring operated at $6 \mathrm{GeV}$ in $7 / 8+1$ filling mode with a maximum electron current of $200 \mathrm{~mA}$. The monochromator was equipped with a pair of He-cooled $\mathrm{Si}(220)$ crystals. Using the fundamental undulator peak, the total incident flux on the sample was $10^{13}$ photons s ${ }^{-1}$ in a spot size of $0.3 \mathrm{~mm} \times 1.2 \mathrm{~mm}$. The incident intensity, monitored by detecting the X-ray scattering from a thin Kapton foil in the incident beam path, and the fluorescence yield from the sample were measured by means of two Si diode detectors. (iii) $\mathrm{V}_{2} \mathrm{O}_{3}, \mathrm{~V}_{2} \mathrm{O}_{4}$ and $\mathrm{V}_{2} \mathrm{O}_{5}$ spectra were collected at the XAFS beamline (Di Cicco et al., 2009) at Elettra Sincrotrone Trieste (Italy). The storage ring operated at $2.4 \mathrm{GeV}$ in top-up mode with a typical current of circa $160 \mathrm{~mA}$. The monochromator was equipped with a pair of $\mathrm{Si}(111)$ crystals. Harmonics were rejected using a pair of Pt-coated mirrors. Spectra were collected in transmission mode and both the incoming and outgoing beams have been measured with ionization chambers. The energy was calibrated in all cases using a metallic vanadium foil. The differences in energy resolution between the different monochromator crystals have been taken into account for computing the broadening factors used in the fit.

Absorption coefficient $\mu$ was extracted from raw data according to the procedures described by Lee et al. (1981). Self-absorption correction has been applied for spectra collected in fluorescence mode by the FLUO algorithm contained in the IFEFFIT (Newville, 2001) package. All the

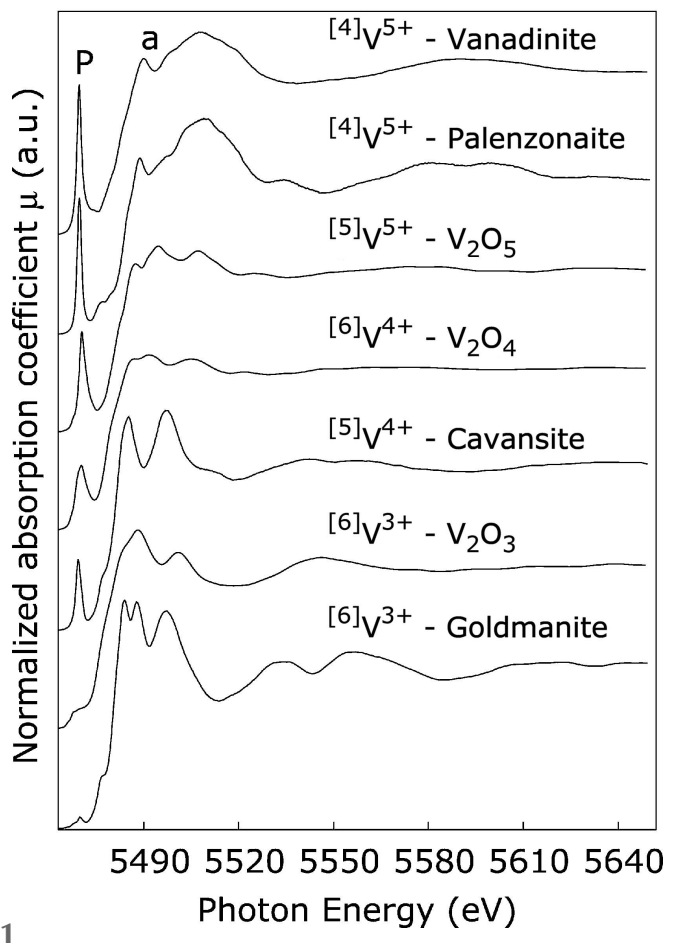

Figure 1

Normalized experimental $\mathrm{V} K$-edge XANES spectra of different vanadium compounds. The normalization procedure is explained in the text. In the figure the pre-edge peak $\mathrm{P}$ and the $1 s \rightarrow 4 p$ peak a are shown. In ${ }^{[x]} \mathrm{V}^{y}, x$ represents the $\mathrm{V}$ coordination geometry and $y$ represents the formal valence. spectra have then been normalized to one at the high-energy side of the spectra. Both self-absorption correction and normalization are achieved using the ATHENA (Ravel \& Newville, 2005) program included in the IFEFFIT (Newville, 2001) package. The experimental XANES spectra are displayed in Fig. 1.

\section{Results and discussion}

The theoretical XANES spectra of the model compounds have been calculated by means of the MXAN (Benfatto \& Della Longa, 2001, 2009; Benfatto et al., 2003) code. The $M X A N$ procedure utilizes the real-space multiple scattering (RSMS) method in the Green function formalism to calculate the final states of the Dyson equation in the framework of the muffin-tin (MT) approximation for the shape of the Coulomb part of the potential. The RSMS algorithm is the one contained in the CONTINUUM (Natoli \& Benfatto, 1986) code. We have used the real Hedin-Lundqvist (Hedin \& Lundqvist, 1971) potential to describe the self-energy. The electronic damping of the XAFS signal is taken into account using a plasmon resonance model, whereas the contribution from thermal damping is neglected. The MT potential is the spherically averaged potential around the atoms and constant in the interstitial regions. The radii of the MT spheres are chosen using the Norman (Norman, 1976) criterion, including a small, partial overlap of the spheres. In this framework it is possible to describe the MT potential using only two free parameters: the MT overlap (ovlp) and the interstitial potential $\left(\mathrm{V}_{\text {imp }}\right)$. The main advantage of this code is the option to fit the structural parameters (distance and angles) and the free theoretical parameters (muffin-tin spheres radii and overlap) with a least-squares method in order to obtain the best agreement with the experimental data. However, it does not use a self-consistent field (SCF) method to calculate the potentials and the charge densities, so that variables such as the interstitial potential must be specified in the fit. This choice is explained by Benfatto \& Della Longa (2009) and it is made to save computational time without losing physical rigour.

The program has been run on a consumer-grade personal computer (Intel Quad Core i7 4770 CPU, 8 GB RAM). The calculations have been distributed on three of the four cores using the parallelization capabilities offered by $M X A N$. Total computing time varied according to the cluster size and $l_{\max }$ value, ranging from around one day to one week.

The calculation of the theoretical spectra and the fit with the normalized experimental one was carried out after the optimization of the number of scatterers (nsca) and the angular momentum cutoff $\left(l_{\max }\right)$. The values are reported in Table 2 . It is known (Wu et al., 1996, 2004; Cabaret et al., 1998) that in order to reproduce the experimental spectra with sufficient accuracy a cluster radius of 6-7 $\AA$ has to be considered. More details, together with details on palenzonaite, as an example, are reported in the supporting information. Once suitable values of nsca and $l_{\max }$ are chosen for the calculation of the theoretical XANES spectra, then we proceed by keeping nsca and $l_{\max }$ fixed and fitting non-structural parameters such as 
Table 2

Overview of the MT parameters computed by MXAN.

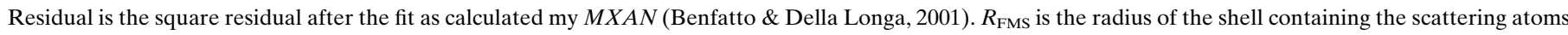

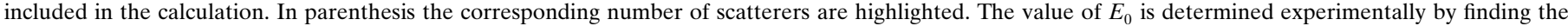

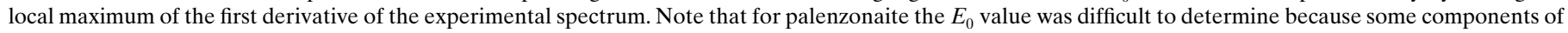

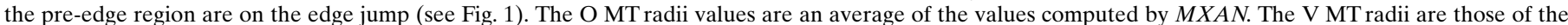
central atom

\begin{tabular}{|c|c|c|c|c|c|c|c|c|c|}
\hline Compound & V type & Residual & $R_{\mathrm{FMS}}(\AA)$ & $l_{\max }$ & MT ovlp & $\mathrm{V}_{\text {imp }}(\mathrm{eV})$ & $E_{0}(\mathrm{eV})$ & $\mathrm{V} R_{\mathrm{MT}}(\AA)$ & $\mathrm{O} R_{\mathrm{MT}}(\AA)$ \\
\hline Goldmanite & ${ }^{[6]} \mathrm{V}^{3+}$ & 9.5 & $6.8(123)$ & 3 & $0.078(5)$ & $-9.3(6)$ & 5475.5 & 1.248 & 0.824 \\
\hline $\mathrm{V}_{2} \mathrm{O}_{3}$ & ${ }^{[6]} \mathrm{V}^{3+}$ & 10.7 & $6.2(110)$ & 4 & $0.07(1)$ & $-14.5(6)$ & 5475.4 & 1.196 & 0.921 \\
\hline Cavansite & ${ }^{[5]} \mathrm{V}^{4+}$ & 1.6 & $6.9(90)$ & 3 & $0.27(2)$ & $-9.9(7)$ & 5476.0 & 1.143 & 0.949 \\
\hline $\mathrm{V}_{2} \mathrm{O}_{4}$ & ${ }^{[6]} \mathrm{V}^{4+}$ & 10.6 & $6.3(102)$ & 4 & $0.14(1)$ & $-17(2)$ & 5478.1 & 1.091 & 0.896 \\
\hline Palenzonaite & ${ }^{[4]} \mathrm{V}^{5+}$ & 3.9 & $6.3(90)$ & 3 & $0.20(1)$ & $-12.5(8)$ & 5482.4 & 1.090 & 0.945 \\
\hline Vanadinite & ${ }^{[4]} \mathrm{V}^{5+}$ & 1.9 & $6.8(90)$ & 4 & $0.21(2)$ & $-13.8(8)$ & 5481.8 & 1.096 & 0.963 \\
\hline $\mathrm{V}_{2} \mathrm{O}_{5}$ & ${ }^{[5]} \mathrm{V}^{5+}$ & 10.6 & 6.9 (119) & 4 & $0.28(2)$ & $-12.3(8)$ & 5480.9 & 1.105 & 1.027 \\
\hline
\end{tabular}

$\mathrm{V} 0_{\text {imp }}$, ovlp and all the broadening and alignment parameters [keeping constant the core-hole broadening to $1.01 \mathrm{eV}$ (literature value; Krause \& Oliver, 1979)]. For some compounds in which the $\mathrm{V}$ crystallographic site has a partial occupation we decided to use a simplified cluster, i.e. we only allowed single occupation by the atom with higher stoichiometry and performed a structural refinement of the first coordination shell. MXAN performs a simplex (known as Nelder-Mead or Amoeba algorithm) fit for the structural and MT parameters, and for every trial it minimizes the broadening and alignment using a Monte Carlo procedure. When the calculation is close enough to the best fit the program proceeds with a least-squares fit to obtain the covariance matrix and from that the error on the fit parameters. The calculation of the absorption cross section is the most timeconsuming part of the procedure because it involves the inversion of a big scattering matrix for every energy point. The use of the Monte Carlo algorithm on the broadening and alignment parameters saves a lot of computational time because it is only applied after the absorption cross section is calculated. The pre-edge part of the experimental XANES spectrum is not calculated due to poor reliability of the MT theory in this region for the systems studied here.

In Fig. 2 it is possible to see an overview of the $M X A N$ fit for the model compounds, whereas the MT parameters obtained by the best fit are summarized in Table 2. There is a general good agreement between the theoretical and the experimental spectra. It can also been seen in both Fig. 2 and Table 2 that there is a very good agreement for the compounds lacking short $\mathrm{V}-\mathrm{V}$ distances, i.e. the minerals goldmanite, palenzonaite, vanadinite and cavansite. On the other hand, for the compounds $\mathrm{V}_{2} \mathrm{O}_{3}, \mathrm{~V}_{2} \mathrm{O}_{4}$ and $\mathrm{V}_{2} \mathrm{O}_{5}$, despite most of the experimental features being well reproduced in the theoretical spectra, some of the intensities or widths still do not match satisfactorily, producing a decrease of the goodness of fit between theory and experiment. We believe the main problems stem from the fact that FMS theory is an independent particle approach, thus neglecting the electron-electron correlation. This may in turn lead to a wrong estimation of the white line and multiple scattering peaks intensities (Farges, 2005; Nakanishi \& Ohta, 2009). So, the relative intensity of the peaks, when many $\mathrm{V}-\mathrm{V}$ multiple scattering phenomena are involved, may not be accurate, especially at lower energy. Whereas only a few systematic studies on V-bearing model compounds have been made so far (Bordage et al., 2010), in the present analysis we provided a set of non-structural parameters which can be useful as starting values in calculating theoretical spectra (see Table 2).

The use of simplified clusters in the refinement of the garnets (goldmanite and palenzonaite) was a necessity of the FMS theory which does not allow for partial site occupation. Furthermore, the crystallographic data available in the literature only account for the average position given by all of the atoms at any given crystallographic site. More noticeable differences can be represented by a site distortion around the central atom (vanadium in our case). We took this effect into account by giving a structural degree of freedom to the system by allowing a $5 \%$ coherent variation $( \pm 0.1 \AA)$ of the oxygen atoms in the first shell.

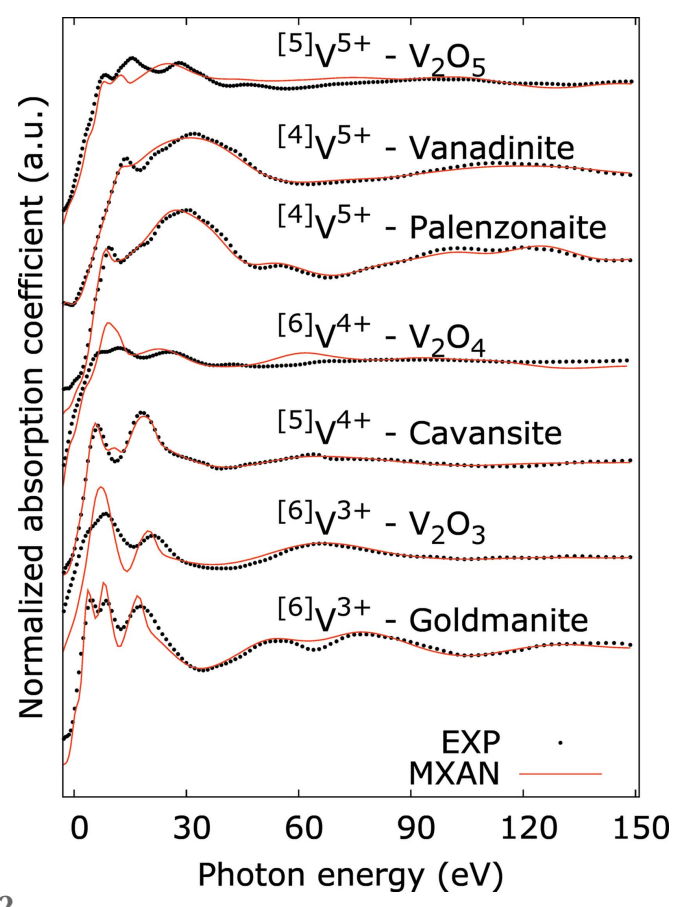

Figure 2

Overview of the MXAN XANES calculations for the set of the model compounds. 
The non-structural parameters resulting from the best fits are listed in Table 2, whereas some relationships between them and ionic radii or edge energies are discussed in the following paragraphs. It has already been reported (Benfatto \& Della Longa, 2009) that the muffin-tin radii of the absorbing atom are proportional to the ionic radii and that the interstitial potential ( $\mathrm{V} 0_{\text {imp }}$ in $M X A N$ nomenclature) is proportional to $E_{0}^{\mathrm{M}}-E_{0}$, where $E_{0}^{\mathrm{M}}$ is the edge energy of the corresponding metal (in the case of $\mathrm{V}$ it is $5465 \mathrm{eV}$ ) (Nakanishi \& Ohta, 2009). Both of these considerations are plausible: the MTradii are calculated by spherically averaging the charge density [Norman criterion (Norman, 1976)] and the ionic radii are calculated in the touching spheres ionic approximation (Shannon, 1976); $\mathrm{V}_{\text {imp }}$ can be seen as the (binding) potential that the photoelectron 'experiences' once ejected from the absorbing atom.

The Shannon ionic radii available in the literature for several $\mathrm{V}$ oxidation states and coordination numbers versus the corresponding muffin-tin radii, as calculated by the $M X A N$ fit of the studied model compounds, are reported in Fig. 3. With the exception of the ${ }^{[6]} \mathrm{V}^{4+}$ case, for which the fit agreement is quite poor, it is possible to notice the trend of MT radius increase at increasing Shannon ionic radii.

In Fig. 4, the plot of $E_{0}^{\mathrm{M}}-E_{0}$ versus $\mathrm{V}_{\text {imp }}$ (only for the four minerals with a more accurate fit and for $\mathrm{V}_{2} \mathrm{O}_{5}$ ) and a linear regression are reported. The reasons for keeping $\mathrm{V}_{2} \mathrm{O}_{3}$ and $\mathrm{V}_{2} \mathrm{O}_{4}$ out of the plot are as follows: (i) for $\mathrm{V}_{2} \mathrm{O}_{3} M X A N$ highly overestimates the intensity of the first peak, and this influences the determination of the $\mathrm{V} 0_{\text {imp }}$ parameter because it influences the relative intensity of the peaks; (ii) $\mathrm{V}_{2} \mathrm{O}_{4}$ was not accurately calculated by $M X A N$ and so the determination of the MT parameters cannot be trusted. Here a trend is also observed, although more model compounds are needed to build a more meaningful relationship. The trend shows a linear-like behaviour. The fit parameters in the equation $f(x)=m x+b$ are $m=$ $0.56 \pm 0.09$ and $b=-4 \pm 1$. Albeit the linearity of the trend

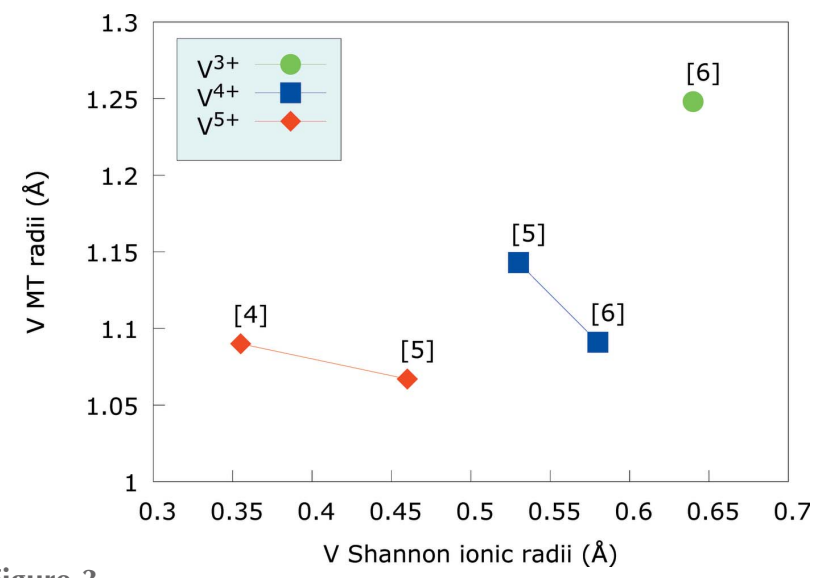

Figure 3

Shannon ionic radii versus MT radii for the different V coordination and oxidation states. The ionic radii are taken from literature data (Shannon, 1976), whereas the MTradii are determined in the present work by means of $M X A N$ fit. For ${ }^{[6]} \mathrm{V}^{3+}$ and ${ }^{[4]} \mathrm{V}^{5+}$ the value for the MT radius is calculated as an average between the two respective structures. The lines are only guides for the eye and connect points representing $\mathrm{V}$ in the same oxidation state.

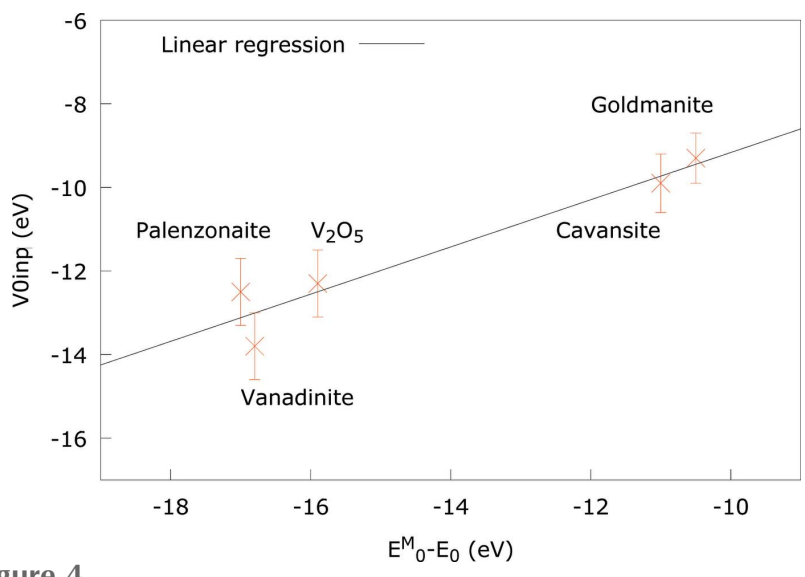

Figure 4

$E_{0}^{\mathrm{M}}-E_{0}$ versus $\mathrm{V} 0_{\text {imp }}$ for the different $\mathrm{V}$ coordinations and oxidation states.

in Fig. 4 cannot be tested, it is clear that the $\mathrm{V} 0_{\text {imp }}$ value decreases with the increase of the edge energy $E_{0}$ of the model compound.

Figs. 3 and 4 thus represent a correlation between physical properties (ionic radii and absorption edges) and non-physical model-dependent parameters (MT radii and interstitial potential). We wish to stress that it is not a way to give a physical meaning to non-physical parameters, but to correlate them to measurable properties and facilitate the study of unknown V-bearing compounds. Whereas in the $\mathrm{V}$ model compounds reported here the structure is reasonably well known, it has been possible to focus the fit of the XANES spectra to derive reliable values of non-structural parameters such as the MT radii and $\mathrm{V} 0_{\text {imp }}$. However, in unknown or poorly known structures (such as glasses or complex systems), the risk of correlations of structural and non-structural parameters in fitting theoretical and experimental spectra is high. Thus, the availability of reliable MT parameters which have been proven good in simulating spectra of $\mathrm{V}$ model compounds can be a great help in the initial steps of theoretical XANES calculations. In this respect, it is also important to observe general trends of the values of MT parameters as a function of the absorber oxidation state and coordination geometry. The data reported here will provide a good starting point for the calculation of $\mathrm{V} K$-edge theoretical XANES spectra for complex systems in order to avoid or, at least, minimize the correlation between variables. A similar analysis involving more $\mathrm{V}$ model compounds is needed in order to test the behaviour of such MT parameters.

\section{Conclusions}

In this work we have applied FMS theory in the MT approximation in a set of V-bearing model compounds: (1) to test the reliability of the theory in calculating the theoretical spectra of the model compounds and (2) to observe trends in the MT parameters as a function of the $\mathrm{V}$ oxidation state and geometry to build a framework that could be used to analyse compounds with unknown structure. We have shown that FMS theory is able to calculate theoretical spectra of V-bearing 
compounds. However, the agreement between the experimental and theoretical spectra is poorer in the case of V-rich compounds $\left(\mathrm{V}_{2} \mathrm{O}_{3}, \mathrm{~V}_{2} \mathrm{O}_{4}\right.$ and $\left.\mathrm{V}_{2} \mathrm{O}_{5}\right)$ possibly because of the $e-e$ interaction that is not taken into account the FMS theory. The non-structural parameters (MT overlap and $\mathrm{V}_{\text {imp }}$ ) resulting from the fit of the experimental spectra of the model compounds can be useful as starting values for the calculation of theoretical spectra when the complexity of the structural refinement by $M X A N$ requires some preliminary assumption.

Even if the model compounds were opportunely chosen to represent most of the common $\mathrm{V}$ oxidation states and coordination geometries, in the future it would be interesting to test the FMS theory on a larger variety of compounds to have a more meaningful comparison and a larger data set of MT parameters.

\section{Acknowledgements}

The authors wish to thank the staff at BM08 and ID26 (ESRF, Grenoble, France) and at XAFS (Elettra, Trieste, Italy) for their kind support and assistance during the experiments. FIRB funds to GG are acknowledged for financial support.

\section{References}

d'Acapito, F. et al. (1998). ESRF Newsl. 30, 42-44.

Benfatto, M. \& Della Longa, S. (2001). J. Synchrotron Rad. 8, 10871094.

Benfatto, M. \& Della Longa, S. (2009). J. Phys. Conf. Ser. 190, 012031.

Benfatto, M., Della Longa, S. \& Natoli, C. R. (2003). J. Synchrotron Rad. 10, 51-57.

Bordage, A., Brouder, C., Balan, E., Cabaret, D., Juhin, A., Arrio, M., Sainctavit, P., Calas, G. \& Glatzel, P. (2010). Am. Miner. 95, 11611171.

Bordiga, S., Groppo, E., Agostini, G., van Bokhoven, J. A. \& Lamberti, C. (2013). Chem. Rev. 113, 1736-1850.

Bunker, G. (2010). Introduction to XAFS: A Practical Guide to X-ray Absorption Fine Structure Spectroscopy. Cambridge University Press.

Cabaret, D., Bordage, A., Juhin, A., Arfaoui, M. \& Gaudry, E. (2010). Phys. Chem. Chem. Phys. 12, 5619-5633.

Cabaret, D., Sainctavit, P., Ildefonse, P. \& Flank, A.-M. (1998). Am. Miner. 83, 300-304.

Chaurand, P., Rose, J., Briois, V., Salome, M., Proux, O., Nassif, V., Olivi, L., Susini, J., Hazemann, J.-L. \& Bottero, J.-Y. (2007). J. Phys. Chem. B, 111, 5101-5110.

Cicco, A. D., Aquilanti, G., Minicucci, M., Principi, E., Novello, N., Cognigni, A. \& Olivi, L. (2009). J. Phys. Conf. Ser. 190, 012043.

Danisi, R., Armbruster, T. \& Lazic, B. (2012). Am. Miner. 97, 18741880.

Faiz, M., Mekki, A., Mun, B. \& Hussain, Z. (2007). J. Electron Spectrosc. Relat. Phenom. 154, 60-62.

Farges, F. (2005). Phys. Rev. B, 71, 155109.

Finger, L. W. \& Hazen, R. M. (1980). J. Appl. Phys. 51, 5362-5367.

Frazier, L. \& France, P. (1977). J. Phys. Chem. Solids, 38, 801-808.

Gauthier, C., Solé, V. A., Signorato, R., Goulon, J. \& Moguiline, E. (1999). J. Synchrotron Rad. 6, 164-166.

Giuli, G., Paris, E., Mungall, J., Romano, C. \& Dingwell, D. (2004). Am. Miner. 89, 1640.

Hävecker, M., Knop-Gericke, A., Mayer, R. W., Fait, M., Bluhm, H. \& Schlögl, R. (2002). J. Electron Spectrosc. Relat. Phenom. 125, 79-87. Hedin, L. \& Lundqvist, B. I. (1971). J. Phys. C, 4, 2064-2083.

Katoh, T., Inda, Y., Baba, M. \& Ye, R. (2010). J. Ceram. Soc. Jpn, 118, 1159-1162.
Kavner, A., Walker, D., Sutton, S. \& Newville, M. (2007). Earth Planet. Sci. Lett. 256, 314-327.

Krause, M. O. \& Oliver, J. H. (1979). J. Phys. Chem. Ref. Data, 8, 329338.

Laufek, F., Skala, R., Haloda, J. \& Cisarova, I. (2006). J. Czech. Geol. Soc. 51, 271-275.

Lee, P. A., Citrin, P. H., Eisenberger, P. \& Kincaid, B. M. (1981). Rev. Mod. Phys. 53, 769-806.

Lee, P. A. \& Pendry, J. B. (1975). Phys. Rev. B, 11, 2795-2811.

McGreevy, R. L. (2001). J. Phys. Condens. Matter, 13, R877-R913.

Moretti, A., Giuli, G., Nobili, F., Trapananti, A., Aquilanti, G., Tossici, R. \& Marassi, R. (2013). J. Electrochem. Soc. 160, A940-A949.

Mott, N. (1968). J. Non-Cryst. Solids, 1, 1-17.

Munakata, M. (1960). Solid State Electron. 1, 159-163.

Nagashima, M. \& Armbruster, T. (2012). Mineral. Mag. 76, 10811097.

Nakanishi, K. \& Ohta, T. (2009). J. Phys. Condens. Matter, 21, 104214.

Natoli, C. \& Benfatto, M. (1986). J. Phys. Colloq. 47, C8-11-C8-23.

Newville, M. (2001). J. Synchrotron Rad. 8, 322-324.

Norman, J. G. (1976). Mol. Phys. 31, 1191-1198.

Ori, G., Montorsi, M., Pedone, A. \& Siligardi, C. (2011). J. Non-Cryst. Solids, 357, 2571-2579.

Pascarelli, S., Boscherini, F., D’Acapito, F., Hrdy, J., Meneghini, C. \& Mobilio, S. (1996). J. Synchrotron Rad. 3, 147-155.

Poumellec, B., Marucco, J. F. \& Touzelin, B. (1987). Phys. Rev. B, 35, 2284-2294.

Prado, R. J. \& Flank, A.-M. (2005). Phys. Scr. T115, 165.

Ravel, B. \& Newville, M. (2005). J. Synchrotron Rad. 12, 537-541.

Rehr, J. \& Ankudinov, A. L. (2005). Coord. Chem. Rev. 249, 131140.

Righter, K., Sutton, S., Danielson, L., Pando, K., Schmidt, G., Yang, H., Berthet, S., Newville, M., Choi, Y., Downs, R. \& Malavergne, V. (2011). Am. Miner. 96, 1278-1290.

Rogers, K. D. (1993). Powder Diffr. 8, 240-244.

Safonova, O., Florea, M., Bilde, J., Delichere, P. \& Millet, J. (2009). J. Catal. 268, 156-164.

Sakurai, Y. \& Yamaki, J. (1988). J. Electrochem. Soc. 135, 791-796.

Sayer, M., Mansingh, A., Reyes, J. \& Rosenblatt, G. (1971). J. Appl. Phys. 42, 2857-2864.

Schindler, M., Hawthorne, F. C. \& Baur, W. H. (2000). Chem. Mater. 12, 1248-1259.

Schuch, M., Christensen, R., Trott, C., Maass, P. \& Martin, S. W. (2012). J. Phys. Chem. C, 116, 1503-1511.

Seshasayee, M. \& Muruganandam, K. (1998). Solid State Commun. 105, 243-246.

Shannon, R. D. (1976). Acta Cryst. A32, 751-767.

Shklover, V., Haibach, T., Ried, F., Nesper, R. \& Novák, P. (1996). J. Solid State Chem. 123, 317-323.

Stizza, S., Davoli, I., Gzowski, O., Murawski, L., Tomellini, M., Marcelli, A. \& Bianconi, A. (1986). J. Non-Cryst. Solids, 80, 175180.

Surnev, S., Ramsey, M. G. \& Netzer, F. P. (2003). Prog. Surf. Sci. 73, 117-165.

Sutton, S., Karner, J., Papike, J., Delaney, J., Shearer, C., Newville, M., Eng, P., Rivers, M. \& Dyar, M. (2005). Geochim. Cosmochim. Acta, 69, 2333-2348.

Taillefumier, M., Cabaret, D., Flank, A.-M. \& Mauri, F. (2002). Phys. Rev. B, 66, 195107.

Trcera, N., Cabaret, D., Rossano, S., Farges, F., Flank, A.-M. \& Lagarde, P. (2009). Phys. Chem. Miner. 36, 241-257.

Walter, A., Herbert, R., Hess, C. \& Ressler, T. (2010). Chem. Cent. J. 4, 3 .

Wong, J., Lytle, F. W., Messmer, R. P. \& Maylotte, D. H. (1984). Phys. Rev. B, 30, 5596-5610.

Wu, Z., Mottana, A., Marcelli, A., Paris, E., Giuli, G. \& Cibin, G. (2004). Phys. Rev. B, 69, 104106.

Wu, Z., Seifert, F., Poe, B. \& Sharp, T. (1996). J. Phys. Condens. Matter, 8, 3323-3336. 